\title{
Validación española de la escala abreviada Five Facet Mindfulness Questionnaire (FFMQ): Un estudio piloto
}

\author{
CRISTIAN COO CALCAGNI \\ coo@uji.es \\ MARISA SALANOVA SORIA \\ salanova@uji.es
}

\section{Resumen}

Introducción: Durante los últimos años se ha probado la eficacia de intervenciones basadas en mindfulness (MBIS) en todo tipo de contextos (clínico, educacional, laboral, etc.). La medida del mindfulness como elemento activo de dichas intervenciones constituye un elemento fundamental para la evaluación de su impacto. El cuestionario Five Facet Mindfulness Questionnaire (FFMQ, Baer y cols., 2008) es una herramienta de evaluación que sintetiza varias formas y constructos de medición utilizados anteriormente en un esfuerzo por desarrollar una medida completa e integradora a nivel teórico y práctico. Este estudio pretende validar una versión abreviada de la escala FFMQ, de 20 ítems mediante un estudio piloto. Método: Se analizaron sus propiedades psicométricas realizando un análisis estadístico de los ítems, un análisis factorial y una estimación de la fiabilidad a través del Alpha de Cronbach. Se utilizó una muestra de 209 trabajadores de organizaciones pertenecientes a las áreas de salud y servicios, la mayoría de ellos sin experiencia previa en mindfulness. Para evaluar la posible esfericidad de los datos obtenidos se aplicaron las pruebas de Kaiser-Meyer-Olkin (KMO) Bartlett. Resultados: El coeficiente alfa de Cronbach fue 0,84. Los resultados de la extracción de factores muestran la agrupación de los 20 ítems seleccionados en 5 factores que explican el $65 \%$ de la varianza. Conclusiones: Los resultados muestran que la escala es válida y fiable para medir la experiencia de mindfulness. Se manifiesta una clara similitud de las propiedades psicométricas de la versión española del FFMQ con la versión original.

Palabras clave: mindfulness, FFMQ, validación, estudio piloto.

\footnotetext{
Abstract

Introduction: During the last ten years the efficacy of Mindfulness Based Interventions has been proven in different contexts (clinical, educational and organizational). The measurement of Mindfulness as the active component of those interventions is a key element for the impact evaluation of those interventions. The Five Facet Mindfulness Questionnaire (FFMQ, Baer y cols. 2008) is an evaluation tool that synthesizes various
} 
forms and constructs of measurement utilized previously in an effort to develop a complete and integrative measurement instrument on both a practical and theoretical level. This study aims to validate an abbreviated version of twenty items based on the original FFMQ scale. Method: Psychometrical properties of the test where observed utilizing statistical analysis of the items, confirmatory factorial analysis, and validity through Cronbach's Alphas. A sample of 209 workers with no prior meditation experience belonging to health and services organizations participated in the study. Results: To evaluate sample adjustment and possible data sphericity KMO Bartlett tests where applied. Cronbach's Alphas was 0,84 , CFA showed a grouping of the twenty selected items in five factors that explain $65 \%$ of the variance. Conclusions: Results indicate that the scale is valid and reliable to measure mindfulness. A clear similitude of psychometrical properties between the Spanish version and the original FFMQ scale can be observed.

Keywords: mindfulness, FFMQ, validation, pilot study.

\section{Introducción}

Mindfulness es una traducción de la palabra, en lengua pali, Sati, traducida al inglés por primera vez en 1881 por T. W. Rhys Davids que se comprende como una adaptación cultural de carácter secular de técnicas y metodologías budistas originadas hace más de 2500 años, cuyo objetivo es el desarrollo del máximo potencial de la mente a través de la práctica regular de la meditación (Trungpa, 1993). Desde la conversación académica contemporánea, ha sido definido como la capacidad inherente a la conciencia humana, que permite atender a los fenómenos que se experimentan en cada momento presente, de un modo en el que se aceptan tal y como emergen en la conciencia, sin realizar juicios sobre ellos (Baer, Smith y Allen, 2004; Brown y Ryan, 2003).

Diversos estudios demuestran los numerosos beneficios para la salud en general y la salud mental en particular, relacionados al mindfulness al ser incorporado como una práctica cotidiana (e.g. Baer y cols., 2008; Brown y Ryan, 2003; Brown, Ryan y Creswell, 2007b; Carlson y Brown, 2005; Walach, Buchheld, Buttenmüller, Kleinknecht, y Schmidt, 2006), lo cual pone en manifiesto su eficacia como herramienta para cultivar el bienestar (Baer, 2003; Baer, y cols., 2008; Germer, 2005; Kabat-Zinn, 2003). Estos hallazgos han alimentado el interés de la psicología occidental por seguir estudiando la naturaleza del constructo y por incorporar diversas técnicas y métodos que desarrollen el mindfulness en sus intervenciones preeminentemente de carácter clínico (Baer, Smith, Hopkins, Krietemeyer y Toney 2006; Mace, 2007).

A pesar de esto, Baer y cols. (2008), Dimidjian y Linehan (2003a), y Kostanski y Hassed (2007) afirmaron que aún son insuficientes los estudios que se han realizado para investigar la naturaleza del mindfulness, su relación con otros constructos psicológicos, las diversas técnicas para desarrollar mayores niveles de mindfulness, los efectos del entrenamiento en mindfulness sobre la salud y, sobre todo, los mecanismos y procesos por los cuales la práctica del mindfulness conlleva a resultados beneficiosos.

En el contexto del desarrollo de intervenciones basadas en mindfulness (MBIS), tanto para hacer frente al manejo a las experiencias de estrés y factores psicosociales que constituyen potenciales daños a las personas, como para mejorar el desempeño óptimo y la salud para promover mayores niveles de calidad de trabajo y excelencia organizacional (Salanova, Llorens, Torrente, y Acosta, 2013) es necesario contar con una herramienta de medición de 
dicho constructo validada en una población que cumpla con las características de los grupos objetivo de los programas de intervención. Esto con la finalidad de clarificar cuales son aquellos procesos mediante los cuales el mindfulness actúa, evaluar las variables sobre las que se espera intervenir y poder observar la efectividad de la intervención. Es por esto que surge esta iniciativa de validación del FFMQ en una población de carácter no clínico y, específicamente, en profesionales de la salud y servicios, grupo objetivo sobre el cual se enfocan las primeras MBI's desarrolladas por el equipo de investigación WONT de la Universitat Jaume I.

Seleccionamos la escala FFMQ dado que ha demostrado tener características psicométricas prometedoras y entendemos que es el mejor instrumento para medir el mindfulness hasta la fecha (May y O'Donovan, 2007), al haber sido creado a partir del análisis factorial de cinco cuestionarios que miden el mindfulness. Ello permitió combinar los ítems más representativos de los distintos instrumentos para formar cinco factores e integrar las distintas definiciones operacionales existentes hasta el momento de su creación (Baer y cols., 2006; Baer y cols., 2008; May y O'Donovan, 2007).

Con todo ello, el objetivo principal de esta investigación es analizar la confiablidad y validez de constructo del FFMQ en un grupo de profesionales no meditadores pertenecientes al sector ocupacional sanitario.

\section{Método}

\section{Muestra y procedimiento}

Se aplicó el cuestionario abreviado FFMQ a una muestra de 227 participantes (86 \% mujeres) pertenecientes a organizaciones de salud y servicios en el contexto de la evaluación de organizaciones saludables y resilientes mediante la herramienta HERO (Healthy and Resilient Organization) desarrollada por el equipo de investigación WONT (Salanova y cols., 2012) en el marco de un proyecto llevado a cabo con el apoyo del Ministerio de Economía para identificar las best practices de aquellas pequeñas y medianas empresas que lograron sobrevivir y proliferar durante el periodo de crisis económica reinante en en los ultimos años en España.

La selección de los participantes se vio influida por la participación de las organizaciones involucradas en el proyecto de investigación HERO, es decir, participaron todos los empleados de aquellas organizaciones participantes del proyecto desde el momento en que se toma la decisión de desarrollar MBIs.

El cuestionario fue aplicado en formato on-line a través de la plataforma web del equipo de investigación WONT, entregando un nombre de usuario y una clave asociada a cada uno de los participantes para permitirle su acceso al cuestionario dentro de un plazo establecido de cinco días desde el envío de la invitación a participar.

\section{Variables}

El instrumento abreviado del FFMQ consta de 20 ítems, subdivididos en cinco categorías correspondientes a los factores propuestos en el modelo original del FFMQ, es decir:

1) Observar (conformado por los ítems 6, 10,12,15 y 18) incluye: observar, notar o atender a las experiencias internas y externas, como sensaciones corporales, cogniciones, emociones, visiones, sonidos, y olores. 
2) Describir (conformado por los ítems 7, 18 y 20) se refiere a nombrar y describir las experiencias observadas con palabras, pero sin establecer un juicio ni un análisis conceptual sobre ellas.

3) Actuar conscientemente (conformado por los ítems 1, 2, 4 y 8 ) envuelve el focalizar la atención conscientemente sobre cada actividad que se realiza en cada momento; y puede ser contrastado con el comportamiento mecánico (comúnmente llamado piloto automático), en donde la atención se focaliza en una situación diferente a la que uno está participando (Baer y cols., 2008).

4) No juzgar la propia experiencia (Conformado por los ítems 5, 14, 16 y 19) incluye tomar una posición no evaluativa frente a las experiencias del momento presente, de modo que éstas son aceptadas y permitidas en la conciencia, sin intentar evitarlas, cambiarlas o escaparse de ellas (Baer y cols., 2004 y Baer y cols., 2008).

5) No reaccionar ante la propia experiencia (conformado por los ítems 3, 9, 11 y 13) se refiere a la tendencia a permitir que los sentimientos y emociones fluyan, sin dejarse «atrapar» o dejarse llevar por ellos (Baer y cols., 2008).

Los participantes indicaron si están de acuerdo con cada ítem a través de una escala Likert con un rango de 0 , si es nunca o muy rara vez cierto, a 6 , si es muy a menudo o siempre cierto.

\section{Análisis de datos}

Se realizaron los análisis de confiabilidad mediante Alpha de Cronbach, correlación ítemfactor, análisis factorial utilizando la metodología de extracción por componentes principales y Kaiser-Meyer-Olkin (KMO) y Bartlett con el paquete estadístico SPPS v 22.

\section{Resultados}

Primero, el resultado de la prueba KMO (ver tabla 1) indica que la correlación entre las variables que el FFMQ mide es medio-alta. Y la prueba de Bartlett indica que el análisis factorial es efectivamente aplicable.

Tabla 1

Prueba de KMO y Bartlett

\begin{tabular}{l|lr}
\hline \multicolumn{2}{l}{ Medida Kaiser-Meyer-Olkin de adecuación de muestreo } & 0,838 \\
\hline \multirow{4}{*}{ Prueba de esfericidad de Bartlett } & Aprox. chi-cuadrado & 1837,725 \\
\cline { 2 - 3 } & gl & 190 \\
\cline { 2 - 3 } & Sig. & 0,000 \\
\hline
\end{tabular}

La consistencia interna se obtuvo a partir del cálculo del Alpha de Cronbach para cada factor de la prueba (ver tabla 2). El valor de dicho estadístico puede variar entre 0 y 1 , donde los valores cercanos a 0,25 indican una baja confiabilidad; los cercanos a 0,50, una fiabilidad 
media o regular; los que superan el 0,75, una confiabilidad aceptable; y aquellos mayores a 0,90, una confiabilidad elevada (Hernández, Fernández y Baptista, 2006). El Alpha de Cronbach para la totalidad de la escala es de 0,84 . Lo que indica una confiabilidad alta para su utilizacion (Cohen, 1988). A pesar de esto, los factores «observar», «describir» y «no reaccionar» ante la propia experiencia demuestran solamente una fiabilidad regular $(\alpha<0,75)$

Tabla 2

Consistencia interna ( $\alpha$ ) de la prueba y sus factores

\begin{tabular}{lcc}
\hline & Numero de Ítems & $\alpha$ \\
\hline Escala completa & 20 & 0,84 \\
Observar & 5 & 0,54 \\
Describir & 3 & 0,43 \\
Actuar conscientemente & 4 & 0,80 \\
No juzgar la propia experiencia & 4 & 0,77 \\
No reaccionar ante la propia experiencia & 4 & 0,58 \\
\hline
\end{tabular}

La observación de las correlación entre ítems y factores (ver tabla 3) nos permtie clarificar la consistencia interna de la prueba al momento de discriminar en puntajes altos y bajos. La mayoría los itemes correlacionaron alta y significativamente con sus respectivos factores.

Tabla 3

Correlaciones ítem-factor

\begin{tabular}{|c|c|c|c|c|c|c|}
\hline & & P6 & P10 & $\mathrm{P} 12$ & P15 & P18 \\
\hline Observar & $\begin{array}{l}\text { Correlación } \\
\text { de Pearson }\end{array}$ & $0,688^{* *}$ & $0,709^{* *}$ & $0,478^{* *}$ & $0,553^{* *}$ & $0,724^{* *}$ \\
\hline & & P7 & P18 & P20 & & \\
\hline \multirow[t]{2}{*}{ Describir } & $\begin{array}{l}\text { Correlación } \\
\text { de Pearson }\end{array}$ & $0,466^{* *}$ & $0,799^{* *}$ & $0,759^{* *}$ & & \\
\hline & & P1 & P2 & P4 & P8 & \\
\hline \multirow[t]{2}{*}{$\begin{array}{l}\text { Actuar cons- } \\
\text { cientemente }\end{array}$} & $\begin{array}{l}\text { Correlación } \\
\text { de Pearson }\end{array}$ & $0,779^{* *}$ & $0,873^{* *}$ & $0,797^{* *}$ & $0,758^{* *}$ & \\
\hline & & P5 & P14 & P16 & P19 & \\
\hline \multirow[t]{2}{*}{$\begin{array}{l}\text { No enjuicia- } \\
\text { miento }\end{array}$} & $\begin{array}{l}\text { Correlación } \\
\text { de Pearson }\end{array}$ & $0,735^{* *}$ & $0,813^{* *}$ & $0,866^{* *}$ & $0,688^{* *}$ & \\
\hline & & P3 & P9 & P11 & P13 & \\
\hline No reactividad & $\begin{array}{l}\text { Correlación } \\
\text { de Pearson }\end{array}$ & $0,490^{* *}$ & $0,774^{* *}$ & $0,641^{* *}$ & $0,735^{* *}$ & \\
\hline
\end{tabular}

**La correlación es significativa en el nivel 0,01 (2 colas). 
Todos los factores se correlacionan de manera significativa entre sí (ver tabla 4), lo que constituye una prueba de la validez de constructo de la escala FFMQ.

Tabla 4

Matriz de correlaciones entre factores

\begin{tabular}{lcccc}
\hline Factores & Describir & $\begin{array}{c}\text { Actuar } \\
\text { conscientemente }\end{array}$ & No enjuiciar & No reaccionar \\
\hline Observar & $0,587^{* *}$ & $0,351^{* *}$ & $0,406^{* *}$ & $0,477^{* *}$ \\
Describir & $0,245^{* *}$ & $0,250^{* *}$ & $0,570^{* *}$ \\
Actuar conscientemente & & $0,602^{* *}$ & $0,201^{* *}$ \\
No enjuiciar & & & $0,256^{* *}$ \\
\hline
\end{tabular}

**a correlación es significativa en el nivel 0,01.

Por último, el análisis factorial utilizando el método de extracción por componentes principales indica que los ítems se distribuyen en una estructura de 5 factores que explican el $65 \%$ de la varianza (ver tabla 5). Lo que es consistente con la estructura del modelo propuesta en otros estudios de validación (Baer y cols., 2012, Cebolla y cols., 2012).

Tabla 5

Análisis factorial

\begin{tabular}{cccc}
\hline \multicolumn{4}{c}{ Sumas de extracción de cargas al cuadrado } \\
\hline Componente & Total & \% de varianza & $\%$ acumulado \\
\hline 1 & 5,879 & 29,397 & 29,397 \\
2 & 3,183 & 15,914 & 45,311 \\
3 & 1,683 & 8,416 & 53,727 \\
4 & 1,194 & 5,969 & 59,695 \\
5 & 1,032 & 5,159 & 64,854 \\
\hline
\end{tabular}

\section{Discusión}

El objetivo de este estudio piloto era explorar las cualidades psicométricas de la escala FFMQ abreviada en una población española de carácter no clínico. Los resultados de los análisis de fiabilidad y factorial apoyan la estructura de cinco factores propuesta por Baer y colegas (2006), en donde los factores "observar», «describir», "actuar con consciencia», «no juzgar la experiencia interna» y «no reaccionar ante la experiencia interna» son elementos de un constructo general de mindfulness.

A diferencia de otros estudios de validación (Baer y cols., 2012, Cebolla y cols., 2012), la variable «observar» sí correlacionó de manera positiva con las otras dimensiones del factor en un grupo de individuos sin experiencia previa en meditación, situación exactamente contraria 
a lo ocurrido en dichos estudios. Esto puede deberse en parte a la consistencia interna apenas adecuada presente en dicha subescala $(\alpha=0,54)$, la que pudo verse afectada por el nivel de compromiso y concentración que demuestran los participantes al contestar el cuestionario de manera on-line.

Nuestro estudio tiene varias limitaciones debido a su carácter exploratorio. Primero, el tamaño de la muestra es moderado. No se realizó una confirmación de la estructura de cinco factores evaluando el ajuste de los datos a diferentes tipos de modelos. También es necesario comparar la escala a otros instrumentos de medición de mindfulness y constructos psicológicos asociados y observar la confiabilidad mediante la aplicación del test-retest.

La mayor contribución de este estudio es proveer una validación de una medida de mindfulness para la población española. Nuestros datos sugieren la posibilidad de utilizar el FFMQ es muestras españolas de carácter no clínico. Lo más relevante es contar con un instrumento de medición de mindfulness sensible a la intervención que incluye cinco habilidades diferentes. Creemos que este cuestionario sera de gran utilidad para medir el efecto de intervenciones basadas en mindfulness en muestras españolas.

En conclusión, el objetivo primario de este estudio era explorar las cualidades psicométricas de la traducción española de la escala FFMQ abreviada. Este objetivo se ha cumplido: el FFMQ abreviado ha probado ser un instrumento efectivo para medir mindfulness en muestras españolas.

\section{Referencias bibliográficas}

Baer, R. (2003). Mindfulness training as a clinical intervention: A conceptual an empirical review. Clinical Psychology: Science and Practice, 10, 125-143.

Baer, R., Smith, G. y Allen, K. (2004). Assessment of mindfulness by self-report: The Kentucky Inventory of Mindfulness Skills. Assessment, 11, 191-206.

Baer, R., Smith, G., Hopkins, J., Krietemeyer, J. y Toney, L. (2006). Using self-report assessment methods to explore facets of mindfulness. Assessment, 13, 27-45.

Baer, R., Smith, G., Lykins, E., Button, D., Krietemeyer, J., Sauer, S., Walsh, E., Duggan, D. y Williams, J. (2008). Construct validity of the Five Facet Mindfulness Questionnaire in meditating and nonmeditating samples. Assessment, 15, 329-342.

Baer, R., Walsh, E., Lykins, E. (2009). Assessment of mindfulness. En F. Diddona (ed.), Clinical Handbook of Mindfulness (pp. 153-168). Nueva York: Springer.

Bishop, S., Lau, M., Shapiro, S., Carlson, L., Anderson, N., Carmody, J., Segal, Z., Abbey, S., Speca, M., Velting, D. y Devins, G. (2004). Mindfulness: a proposed operational definition. Clinical Psychology: Science and Practice, 11, 230-241.

Brown, K. y Ryan, R. (2003). The benefits of being present: Mindfulness and its role in psychological well-being. Journal of Personality and Social Psychology, 84, 822-848.

Brown K. y Ryan, R. (2004). Perils and promise in defining and measuring mindfulness: Observations from experience. Clinical Psychology: Science and Practice, 11, 242-248.

Brown, K., Ryan, R. y Creswell, J. (2007a). Addressing Fundamental Questions About Mindfulness. Psychological Inquiry, 18, 272-281.

Brown, K., Ryan, R. y Creswell, J. (2007b). Mindfulness: Theoretical Foundations and Evidence for its Salutary Effects. Psychological Inquiry, 18, 211-237.

Cebolla, A., García-Palacios, A., Soler, J., Guillén, V., Baños, R. y Botella, C. (2012). The European Journal of Psychiatry, 26, 118-126.

Cohen, J. (1988). Statistical power analysis for the behavioral sciences (2nd ed.). Hillsdale, NJ: Lawrence Earlbaum Associates. 
Dimidjian, S. y Linehan, M. M. (2003a). Defining and agenda for future research on the clinical application of mindfulness practice. Clinical Psychology: Science and Practice, 10, 166171.

Dimidjian, S. y Linehan, M. M. (2003b). Mindfulness practice. En W. O’Donohue, J. E. Fisher y S. C. Hayes (eds.), Empirically supported techniques of cognitive behavior therapy: A step-by-step guide for clinicians (pp. 229-237). Nueva York: John Wiley.

Germer, C. K. (2005). Mindfulness. What is it? What does it matter? En C. K. Germer, R. D. Siegel y P. R. Fulton (eds.), Mindfulness and Psychotherapy (pp. 3-27). Nueva York: Guilford Press.

Hernández, R.; Fernández, C. y Baptista, P. (2003). Metodología de la investigación. México: McGraw-Hill.

Kabat-Zinn, J. (2003). Mindfulness-based interventions in context: past, present and future. Clinical Psychology: Science and Practice, 10, 144-156.

Kostanski, M. y Hassed, C. (2007). Mindfulness as a concept and a process. Australian Psychologist, 43, 15-21.

May, S. y O'Donovan (2007). The advantages of the mindful therapist. Psychotherapy in Australia, 13, 46-53.

Salanova, M., Llorens, S., Torrente, P. y Acosta, H. (2013). Intervenciones positivas para potenciar organizaciones saludables y resilientes. En Palací Descals, F. y Bernabé Castaño, M. Consultoría Organizacional, 7, pp. 135-166.

Salanova, M., Llorens, S., Cifre, E. y Martínez, I. (2012). We need a Hero! Toward a Validation of the Healthy Resilient Organization Model. Group \& Organization Management, 37, 758-882.

Trungpa, C. (1993). Training the mind and cultivating loving kindness. Boston y Londres: Shambala.

Walach, H., Buchheld, N., Buttenmüller, V., Kleinknecht, N. y Schmidt, S. (2006). Measuring mindfulness-The Freiburg Mindfulness Inventory (FMI). Personality and Individual Differences, 40, 1543-1555. 